\title{
Spatial filter decomposition for interference mitigation
}

\author{
Rabah Maoudj ${ }^{1 *}$, Michel Terre ${ }^{1}$, Luc Fety ${ }^{1}$, Christophe Alexandre ${ }^{1}$ and Philippe Mege ${ }^{2}$
}

\begin{abstract}
This paper presents a two-part decomposition of a spatial filter having to optimize the reception of a useful signal in the presence of an important co-channel interference level. The decomposition highlights the role of two parts of the filter, one devoted to the maximization of the signal to noise ratio and the other devoted to the interference cancellation. The two-part decomposition is used in the estimation process of the optimal reception filter. We propose then an estimation algorithm that follows this decomposition, and the global spatial filter is finally obtained through an optimal-weighted combination of two filters. It is shown that this two-component-based decomposition algorithm overcomes other previously published solutions involving eigenvalue decompositions.
\end{abstract}

Keywords: SIMO receiver; Optimum combining; Maximum ratio combining; Antenna array

\section{Introduction}

Increasing capacity demand for wireless communication networks should lead to a high co-channel interference level in the future. This interference problem is not new and has been addressed, for a long time, in wireless networks. The first solutions, coming from $2 \mathrm{G}$ networks, were based on frequency reuse patterns while, a few years later, scrambling codes were used, for the same purpose, in $3 \mathrm{G}$ networks. Nowadays, diversity techniques are more and more considered as one of the best answer for this interference problem, especially in 4G networks. The work presented in this paper was initially based on private mobile radio (PMR) characteristics linked to the TETRA enhanced data service (TEDS) standard, but it is suitable also in the context of $4 \mathrm{G}$ transmissions which are based on the Long-Term Evolution (LTE) standard. The presented work addresses essentially the problem of transmission with high interference level ratio and fast varying propagation channels.

In such a context, several previous works have treated and analyzed the best spatial filter to maximize the signal to interference plus noise ratio (SINR). The optimum combining (OC) filter [1-5] can be proposed as an exploitable solution. It was also proven that, in the absence of interference, this optimum combining

\footnotetext{
* Correspondence: rabamail@yahoo.com

${ }^{1}$ CNAM/CEDRIC/Laetitia, Paris, France

Full list of author information is available at the end of the article
}

filter converges to the well-known maximum ratio combining (MRC) filter [6].

The main problem, in the estimation of the optimum combiner filter, resides in the estimation of the covariance matrix of the interference plus noise in addition to the estimation of the useful user propagation channel. The minimum mean square error estimation (MMSE) criterion can be used to estimate the desired user propagation channel, while the covariance matrix can be estimated by the sample matrix inversion (SMI) approach [7-9]. However, this strategy is suboptimal and requires a large signal sample set to get a correct smoothing stage $[10,11]$. Another problem resides in the covariance matrix itself which will be obviously interpolated to the data locations since it is estimated on the pilot locations [10].

In this paper, we will prove that under certain conditions, the OC filter can perfectly be decomposed in two independent components. Hence, when the number of interferers is smaller than the number of receiving antennas, the OC filter can be split in two weighted components, namely MRC filter and interference canceller combiner (ICC). Moreover, for its own scientific interest, it will be proven that this decomposition leads to a new and efficient optimum filter estimation algorithm.

More precisely, we will show that for the MRC part of the OC filter, the classical MMSE can be proposed in order to obtain the estimation of the useful user propagation channel. On the other hand, we will show that the ICC part 
can be estimated by modifying the matched desired impulse response (MDIR) algorithm [12]. This algorithm requires a null linear system solving, and a constraint is required to prevent the trivial solution. Following [12], a constraint, so-called maximum SINR constraint (MSINRC), can be introduced; it will maximize the SINR at the output of the filter. The final solution-vector will then be given by the eigenvector corresponding to the lowest eigenvalue of a Hermitian matrix generated by the algorithm. Due to the presence of the noise, the estimation still remains suboptimal and the result is corrupted by the training noise estimation. It was proven in [13] that combining all eigenvectors, weighted by the inverse of their corresponding output SINR, could lead to an enhanced algorithm, so-called solution-vectors maximum ratio combining (SoMRC). It was shown that this approach gives better performance than the single solution-vector $[13,14]$. Obviously, such kind of algorithms is based on a complex eigendecomposition. To simplify this step, we propose to introduce another constraint, leading to a less complex algorithm where we can avoid the eigendecomposition. We will also show that this new constraint keeps the performance of the algorithm comparable to that of the SoMRC.

The contribution of the presented work in the study of the optimum combiner filter consists on identifying the cases where it can be decomposed to a MRC plus a ICC independent filters. Another contribution consists of the modified MDIR algorithm and in the introduction of the new constraint that does not degrade performances of the SoMRC. A 16-bit digital signal processor (DSP) implementation is also presented in order to evaluate the rounding errors effect on the performance of the algorithm and to determine the possibility of the algorithm execution under some real-time constraints.

The paper is organized as follows. The system model is given in Section 2. Section 3 presents the split of the $\mathrm{OC}$ into two weighted independent parts, namely MRC and ICC. In Section 4, the estimation algorithm for ICC is detailed. A simplification of this estimation algorithm is introduced in Section 5. Numerical results and performance of the algorithms are presented in Section 6. A performance degradation study, due to a practical 16-bit fixed point DSP implementation is detailed in Section 7. Conclusion summarizes the present work in Section 8.

\subsection{Notation}

Vectors and matrices are boldface small and capital letters; the transpose, complex conjugate transpose, and inverse of matrix $A$ are denoted by $A^{T}, A^{H}$, and $A^{-1}$, respectively.

The norm of vector $a$ and the diagonal matrix with the diagonal element extracted from $\boldsymbol{a}$ are denoted respectively by $\|\mathbf{a}\|$ and $\operatorname{diag}\{\mathbf{a}\}, \boldsymbol{I}_{\mathrm{N}}$ is the $N \times N$ identity matrix, and $E[$.$] denotes the statistical expectation.$

\section{System model}

We consider a SIMO structure having $M$ receiving antennas, with one desired signal and $U$ interferers, as presented in Figure 1. Desired user and interferers are transmitting orthogonal frequency-division multiplexing (OFDM) waveforms, and for the sake of simplicity, we will consider that all users are time and frequency synchronized. This remark is not restrictive, and all results presented are not linked to this hypothesis that will nevertheless simplify some notations in the sequel of the paper.

The OFDM frame of the desired user is composed of $K$ subcarriers and $N_{s}$ OFDM symbols. A $N_{g}$ length cyclic prefix is inserted. It is assumed that this cyclic prefix is sufficient to totally suppress intersymbol interferences. We will focus on one OFDM symbol, and we will avoid indicating the symbol number in the notations. Algorithms presented will then be able to cope with a unique OFDM symbol and therefore able to deal with very high-speed propagation channels.

With this assumption, the received sample, after the OFDM demodulation, corresponding to the $k$ th subcarrier on the $m$ th antenna, as presented in Figure 2, can be modeled by Equation 1:

$$
y_{k}(m)=h_{x, k}(m) x_{k}+n_{k}^{I}(m)
$$

$n_{k}^{I}(m)$ denotes an additive noise plus interference term and can be developed as follows

$$
n_{k}^{I}(m)=\sum_{u=1}^{U} h_{z, k, u}(m) z_{k, u}+n_{k}(m)
$$

$Z_{k, u}$ is the symbol transmitted by the $u$ th interferer.

$h_{z, k, u}(m)$ denotes the frequency response of the channel between the $u$ th interferer and the $m$ th antenna. $n_{k}(m)$ represents a centered additive white Gaussian noise term with a variance equals to $\sigma n^{2}$.

$\sum_{u=1}^{U} h_{z, k, u}(m) z_{k, u}$ is then the total contribution of the $U$ interferers received by the $m$ th antenna on the $k$ th subcarrier.

In this paper, we have to identify a spatial filter $w_{k}$ able to estimate the QAM symbol $\hat{x}_{k}$ transmitted by the desired user. This leads to

$$
\hat{x}_{k}=\boldsymbol{w}_{k}^{H} \boldsymbol{y}_{k}
$$

In this equation, corresponding to the $k$ th subcarrier, $\boldsymbol{w}_{k}=\left(\begin{array}{c}w_{k}(0) \\ \vdots \\ w_{k}(M-1)\end{array}\right)$ denotes, as illustrated in Figure 3, $M$ weights of the spatial filter and $\boldsymbol{y}_{k}=\left(\begin{array}{c}y_{k}(0) \\ \vdots \\ y_{k}(M-1)\end{array}\right)$ stands for the $k$ th post-fast Fourier transform (FFT) outputs 


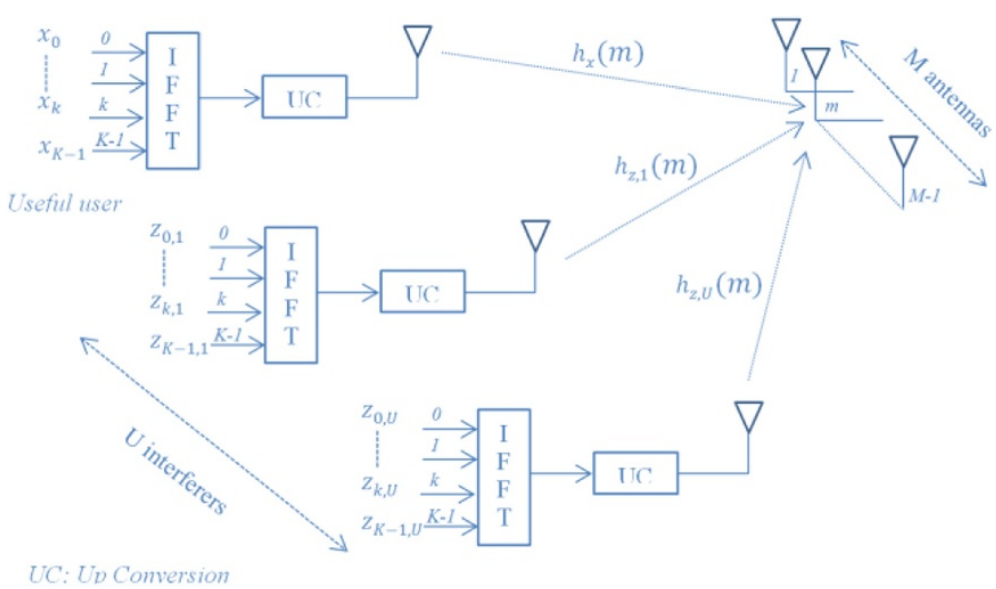

Figure 1 Context of the work, one useful user, and $U$ interferers.

of $M$ antennas. Combining Equations 1, 2, and 3 leads to Equation 4

$$
\hat{x}_{k}=\boldsymbol{w}_{k}^{H}\left(\boldsymbol{h}_{x, k} x_{k}+\sum_{u=1}^{U} \boldsymbol{h}_{z, k, u} z_{k, u}+\boldsymbol{n}_{k}\right)
$$

where $\boldsymbol{h}_{x, k}=\left(\begin{array}{c}h_{x, k}(0) \\ \vdots \\ h_{x, k}(M-1)\end{array}\right)$ denotes the $M$ desired propagation channel frequency responses of the $k$ th subcarrier,

$$
\boldsymbol{h}_{x, k, u}=\left(\begin{array}{c}
h_{x, k, u}(0) \\
\vdots \\
h_{x, k, u}(M-1)
\end{array}\right) \text { the } M \text { interfering propaga- }
$$

tion channel frequency responses between the $u$ th interferer and the receiving antennas, and finally $\boldsymbol{n}_{k}=\left(\begin{array}{c}n_{k}(0) \\ \vdots \\ n_{k}(M-1)\end{array}\right)$ stands for the received noise on the $M$ receiving antennas.

The spatial filter that maximizes the signal to interference plus noise ratio $\left(\operatorname{SINR}_{k}\right)$ for $\hat{x}_{k}$ was introduced in
[1] and studied in [2,3]. Main steps of this derivation are presented hereafter.

We could consider, without loss of generality, that $E\left[\left|x_{k}\right|^{2}\right]=1$ and $E\left[\left|z_{k, u}\right|^{2}\right]=1$. According to Equation 4, the $\mathrm{SINR}_{k}$, at the output of the spatial filter, is then given by

$$
\operatorname{SINR}_{k}=\frac{\boldsymbol{w}_{k}^{H} \boldsymbol{h}_{x, k}^{*} \boldsymbol{h}_{x, k}^{T} \boldsymbol{w}_{k}}{\boldsymbol{w}_{k}^{H} \boldsymbol{R}_{n n, k} \boldsymbol{w}_{k}}
$$

In this equation, $\boldsymbol{R}_{n n, k}$ stands for the $M \times M$ spatial covariance matrix of interference plus noise, and it is expressed by

$$
\boldsymbol{R}_{n n, k}=E\left[\left(\sum_{u=1}^{U} \boldsymbol{h}_{z, k, u} z_{k, u}+\boldsymbol{n}_{k}\right)\left(\sum_{u=1}^{U} \boldsymbol{h}_{z, k, u} z_{k, u}+\boldsymbol{n}_{k}\right)^{H}\right]
$$

Considering the decorrelation between interferer signals and noise terms, we have

$$
\boldsymbol{R}_{n n, k}=\sum_{u=1}^{U} E\left[\boldsymbol{h}_{z, k, u}^{*} \boldsymbol{h}_{z, k, u}^{T}\right]+\sigma_{n}^{2} \boldsymbol{I}_{M}
$$

By introducing the $\boldsymbol{R}_{z z, k}$ matrix defined by

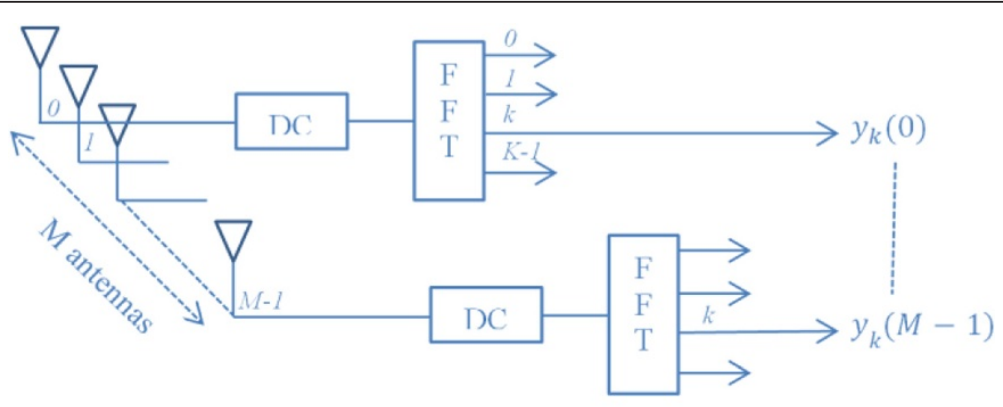

DC: Down Conversion

Figure 2 Receiving structure in the SIMO context. 


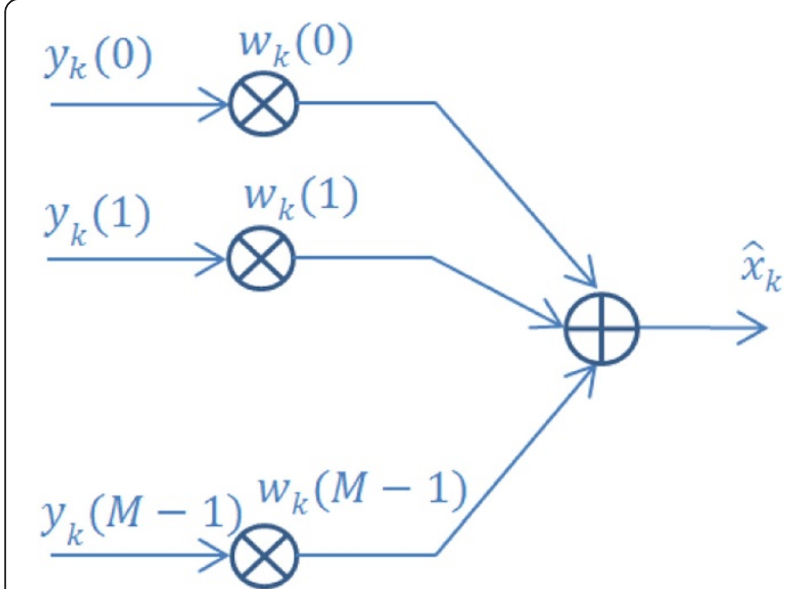

Figure 3 Receiver spatial filter for the $k$ th subcarrier.

$$
\boldsymbol{R}_{z z, k}=\sum_{u=1}^{U} E\left[\boldsymbol{h}_{z, k, u}^{*} \boldsymbol{h}_{z, k, u}^{T}\right]
$$

we can rewrite $\boldsymbol{R}_{n n, k}$ as a summation of two parts as given hereafter

$$
\boldsymbol{R}_{n n, k}=\boldsymbol{R}_{z z, k}+\sigma_{n}^{2} \boldsymbol{I}
$$

At the output of the combiner, in order to perfectly equalize the useful channel, we propose to introduce the following constraint

$$
\boldsymbol{w}_{k}^{H} \boldsymbol{h}_{x, k}^{*}=1
$$

Merging Equations 5 and 9, the $\operatorname{SINR}_{k}$ becomes

$$
\operatorname{SINR}_{k}=\frac{1}{\boldsymbol{w}_{k}^{H} \boldsymbol{R}_{n n, k} \boldsymbol{w}_{k}}
$$

The optimum spatial filter vector $\boldsymbol{w}_{k}$ that maximizes the $\mathrm{SINR}_{k}$ can then be obtained by minimizing $\boldsymbol{w}_{k}^{H} \boldsymbol{R}_{n n, k}$ $\boldsymbol{w}_{k}$ under the constraint $\boldsymbol{w}_{k}^{H} \boldsymbol{h}_{x, k}^{*}=1$. Using Lagrange multiplier, the optimum spatial filter is obtained by solving the following equation

$$
\boldsymbol{w}_{k}=\arg \min \mathcal{L}\left(\frac{1}{\operatorname{SINR}_{k}}, \boldsymbol{w}_{k}, \lambda\right)
$$

where the Lagrangian is defined such that

$$
L\left(\frac{1}{\operatorname{SINR}_{k}}, \boldsymbol{w}_{k}, \lambda\right)=\boldsymbol{w}_{k}^{H} \boldsymbol{R}_{n n, k} \boldsymbol{w}_{k}-\lambda\left(\boldsymbol{w}_{k}^{H} \boldsymbol{h}_{x, k}^{*}-1\right)
$$

Under the assumption that $\operatorname{det}\left(\frac{d^{2} \mathcal{L}\left(\frac{1}{\operatorname{SNIR}_{k}}, \boldsymbol{w}_{k}, \lambda\right)}{d \boldsymbol{w}_{k}^{2}}\right)>0$, the solution is obtained by nulling the derivative of $\mathcal{L}\left(\frac{1}{\operatorname{SNIR}_{k}}, \boldsymbol{w}_{k}, \lambda\right)$ with respect to $\boldsymbol{w}_{k}$

$$
\frac{d L\left(\frac{1}{\operatorname{SINR}_{k}}, \boldsymbol{w}_{k}, \lambda\right)}{d \boldsymbol{w}_{k}}=0
$$

Solving Equation 14 leads to

$$
\boldsymbol{R}_{n, k} \boldsymbol{w}_{k}-\lambda \boldsymbol{h}_{x, k}^{*}=0
$$

The optimal [1] solution is then expressed by

$$
\boldsymbol{w}_{k}=\lambda \boldsymbol{R}_{n n, k}^{-1} \boldsymbol{h}_{x, k}^{*}
$$

The minimum of the solution (Equation 12) is justified by the positive Hermitian characteristic of the covariance matrix $R_{n n, k}$. We have

$$
\operatorname{det}\left(\frac{d^{2} \mathrm{~L}\left(\frac{1}{\operatorname{SINR}_{k}}, \boldsymbol{w}_{k}, \lambda\right)}{d \boldsymbol{w}_{k}^{2}}\right)=\operatorname{det}\left(\boldsymbol{R}_{n n, k}\right)
$$

As $\operatorname{det}\left(\boldsymbol{R}_{n n, k}\right)$ is equal to the product of eigenvalues of $\boldsymbol{R}_{n n, k}$, we have $\operatorname{det}\left(\boldsymbol{R}_{n n, k}\right)>0$. Finally, $\boldsymbol{w}_{k}^{H} \boldsymbol{h}_{x, k}^{*}=1$ leads to

$$
\lambda=\frac{1}{\boldsymbol{h}_{x, k}^{H} \boldsymbol{R}_{n n, k}^{-1} \boldsymbol{h}_{x, k}}
$$

Therefore,

$$
\boldsymbol{w}_{k}=\frac{\boldsymbol{R}_{n, k}^{-1} \boldsymbol{h}_{x, k}^{*}}{\boldsymbol{h}_{x, k}^{H} \boldsymbol{R}_{n n, k}^{-1} \boldsymbol{h}_{x, k}}
$$

Identifying the spatial filter $\boldsymbol{w}_{k}$ through Equation 19 is a very complex task involving $\boldsymbol{R}_{n n, k}$ estimation and inversion plus $\boldsymbol{h}_{x, k}$ estimation. In order to address this spatial filter estimation problem, we propose to split it on two separated filters. This approach highlights how the optimal spatial filter works.

\section{Spatial filter decomposition}

We consider the eigenvector matrix $\boldsymbol{U}_{k}$ and the diagonal matrix $\boldsymbol{I}_{\mu k}$ of the eigenvalues defined such that $\boldsymbol{I}_{\mu k}=\left(\begin{array}{ccc}\mu_{0, k} & 0 & 0 \\ 0 & \ddots & 0 \\ 0 & 0 & \mu_{M-1, k}\end{array}\right)$. Then, the eigenspace decomposition of $\boldsymbol{R}_{z z, k}$ can be written as follows

$$
\boldsymbol{R}_{z z, k}=\boldsymbol{U}_{k} \boldsymbol{I}_{\mu_{k}} \boldsymbol{U}_{k}^{H}
$$

According to the $\boldsymbol{R}_{n n, k}$ decomposition, presented in Equations 7 and 8, we obtain

$$
\boldsymbol{R}_{n n, k}=\boldsymbol{U}_{k} \boldsymbol{I}_{\mu_{k}+\sigma_{n}^{2}} \boldsymbol{U}_{k}^{H}
$$

With $\boldsymbol{I}_{\mu k+\sigma_{n}^{2}}=\boldsymbol{I}_{\mu k}+\sigma_{n}^{2} \boldsymbol{I}_{M}$, we introduce then $\boldsymbol{C}_{n n, k}$ as the transpose adjugate (cofactor) matrix of $\boldsymbol{R}_{n n}$, defined as follows 


$$
\boldsymbol{R}_{n n, k}^{-1}=\frac{\boldsymbol{C}_{n n, k}}{\operatorname{det}\left(\boldsymbol{R}_{n n, k}\right)}
$$

Replacing $\boldsymbol{R}_{n n, k}^{-1}$ by $\frac{\boldsymbol{C}_{n n, k}}{\operatorname{det}\left(\boldsymbol{R}_{n, k}\right)}$ in the denominator of Equation 19 leads to

$$
w_{k}=\frac{1}{\boldsymbol{h}_{x, k}^{H} \boldsymbol{C}_{n n, k} \boldsymbol{h}_{x, k}} \operatorname{det} \boldsymbol{R}_{n n, k} \boldsymbol{R}_{n n, k}^{-1} \boldsymbol{h}_{x, k}^{*}
$$

Knowing that $\operatorname{det}\left(\boldsymbol{R}_{n n, k}\right)=\prod_{i=1}^{M-1}\left(\mu_{i, k}+\sigma_{n}^{2}\right)$, we can write

$$
\operatorname{det}\left(\boldsymbol{R}_{n n, k}\right) \boldsymbol{R}_{n n, k}^{-1}=\boldsymbol{U}_{k} \underline{I}_{\frac{\prod_{\left.\mu+\sigma_{n}^{2}\right)}}{\mu+\sigma_{n}^{2}}} \boldsymbol{U}_{k}^{H}
$$

In this last equation, $\frac{I \prod\left(\mu+\sigma_{n}^{2}\right)}{\mu+\sigma_{n}^{2}}$ stands for a diagonal matrix such that its $m$ th component is given by

$$
\frac{\prod_{i=0}^{M-1}\left(\mu_{i, k}+\sigma_{n}^{2}\right)}{\mu_{m, k}+\sigma_{n}^{2}}=\prod_{i=0}^{i \neq m}{ }^{M-1}\left(\mu_{i, k}+\sigma_{n}^{2}\right)
$$

Then, Equation 24 can be rewritten as follows

$$
\operatorname{det} R_{n n, k} R_{n n, k}^{-1}=C_{z z, k}+\sigma_{n}^{2(M-1)} I+G_{m, k}
$$

where $\boldsymbol{G}_{m, k}$ is null matrix for $M \leq 2$ otherwise is defined by the following expression

$$
\begin{aligned}
G_{m, k}= & \sum_{m_{1}=0}^{M-2} \\
& \times\left((-1)^{m_{1}} R_{z z, k}^{m_{1}} \sum_{m_{2}=1}^{M-m_{1}-1}\left(\sigma_{n}^{2\left(M-m_{2}-1\right)}\left(\sum_{i=1}^{M-1} \mu_{i, k}\right)^{m_{2}-1}\right)\right)
\end{aligned}
$$

As shown in the previous equation, $\boldsymbol{G}_{m, k}$ is linked to the interference covariance matrix. We note that in the case of totally decorrelated interferers, $\boldsymbol{R}_{z z, k}=\sigma_{c}^{2} \boldsymbol{I}$.

Finally, we obtain the following decomposition of the spatial filter $\boldsymbol{w}_{k}$

$$
w_{k}=\frac{1}{h_{x, k}^{H} C_{n n, k} h_{x, k}}\left(C_{z z, k} h_{x, k}^{*}+\sigma_{n}^{2(M-1)} h_{x, k}^{*}+G_{m, k} h_{x, k}^{*}\right)
$$

We conclude that the optimal spatial filter is a combination of three filters: mainly a filter dedicated exclusively to the interference, a maximum ratio combiner filter, and a filter linked to the statistical dependency of the interferers. Due to the complexity of the third filter, the estimation of the optimal spatial filter through the separate estimation of the filters is only interesting in the case of a two-antenna receiver $\left(\boldsymbol{G}_{m, k}=\mathbf{0}\right)$.

\subsection{Analysis for $M=2$}

If we consider now a two-antenna spatial filter $(M=2)$, in this case, the optimal spatial filter given by Equation 27 becomes

$$
w_{k}=\frac{1}{h_{x, k}^{H} C_{n n, k} h_{x, k}}\left(C_{z z, k} h_{x, k}^{*}+\sigma_{n}^{2} h_{x, k}^{*}\right)
$$

With $C_{z z, k} \neq \mathbf{0}$ for $U \geq M-1$.

From Equation 24, we have

$$
C_{n n, k}=U_{k} I_{\frac{\prod\left(\mu+\sigma_{n}^{2}\right)}{\mu+\sigma_{n}^{2}}} U_{k}^{H}
$$

and

$$
C_{n n, k}=C_{z z, k}+\sigma_{n}^{2} I_{M}
$$

Replacing Equation 30 in Equation 28, we obtain

$$
w_{k}=\frac{1}{h_{x, k}^{H} C_{z z, k} h_{x, k}+\sigma_{n}^{2} h_{x, k}^{H} h_{x, k}}\left(C_{z z, k} h_{x, k}^{*}+\sigma_{n}^{2} h_{x, k}^{*}\right)
$$

We introduce now the $\rho_{k}$ scalar defined as follows

$$
\rho_{k}=\frac{h_{x, k}^{H} C_{z z, k} h_{x, k}}{h_{x, k}^{H} h_{x, k}}
$$

After some derivations, we arrive to

$$
w_{k}=\frac{1}{\rho_{k}+\sigma^{2}}\left(\rho_{k} \frac{C_{z z, k} h_{x, k}^{*}}{h_{x, k}^{H} C_{z z, k} h_{x, k}}+\sigma_{n}^{2} \frac{h_{x, k}^{*}}{h_{x, k}^{H} h_{x, k}}\right)
$$

This equation can be presented as follows

$$
w_{k}=\frac{1}{\rho_{k}+\sigma^{2}}\left(\rho_{k} w_{z, k}+\sigma_{n}^{2} w_{h, k}\right)
$$

with

$$
w_{z, k}=\frac{C_{z z, k} h_{x, k}^{*}}{h_{x, k}^{H} C_{z z, k} h_{x, k}}
$$

and

$$
w_{h, k}=\frac{h_{x, k}^{*}}{h_{x, k}^{H} h_{x, k}}
$$

Equation 34 expresses the optimum combiner $\boldsymbol{w}_{k}$ through a weighted combination of two combiners $\boldsymbol{w}_{z, k}$ and $\boldsymbol{w}_{h, k}$.

In this two-part decomposition, $\boldsymbol{w}_{z, k}$ represents the optimum combiner in the case of a noiseless transmission with interference and $\boldsymbol{w}_{h, k}$ represents the maximum ratio combiner $\rho_{k}$ and $\sigma_{n}^{2}$ are two positive scalars representing the degree of contribution of these two combiners $\boldsymbol{w}_{z, k}$ and $\boldsymbol{w}_{h, k}$. 
From this observation, we can derive the following theorem.

Theorem: In the case of two-antenna SIMO receiver, the optimum combiner $\boldsymbol{w}_{k}$ can be expressed as a weighted combination of the optimum combiner $\boldsymbol{w}_{z, k}$ obtained in the case of null additive white Gaussian noise and the maximum ratio combiner $\boldsymbol{w}_{h, k}$.

Furthermore, of the physical interpretation of the optimal spatial filter, this decomposition can also be used to estimate the optimal spatial filter in two steps: a step devoted to the optimum combiner without taking into account the additive noise $\boldsymbol{w}_{z, k}$ and the other to the maximum ratio combiner $\boldsymbol{w}_{h, k}$.

\section{Spatial filter estimation}

\subsection{Estimation algorithm for $w_{h, k}$}

The $\boldsymbol{w}_{h, k}$ filter does not depend on interference but only on the desired signal propagation channel. Its estimation is then directly linked to the estimation of the propagation channel $\boldsymbol{h}_{x, k}$.

In this work, we consider that propagation channels, i.e., useful and interference channels, can be modeled as $L$ taps finite impulse response filters and with $L<K$. We introduce then these impulse responses, corresponding to the $m$ th antenna, through the following vectors

$$
a_{x}(m)=\left(\begin{array}{c}
a_{x, 0}(m) \\
\vdots \\
a_{x, L-1}(m)
\end{array}\right)
$$

Thanks to this hypothesis we can introduce the $\boldsymbol{h}_{x}(m)$ vector that represents the frequency response of the propagation channel between the useful user and the $m$ th antenna

$$
h_{x}(m)=\left(\begin{array}{c}
h_{x, 0}(m) \\
\vdots \\
h_{x, K-1}(m)
\end{array}\right)
$$

The two $\boldsymbol{a}_{x}(m)$ and $\boldsymbol{h}_{x}(m)$ vectors are linked by the following equation

$$
h_{x}(m)=F_{K, L} a_{x}(m)
$$

where $\boldsymbol{F}_{K, L}$ is the $K \times L$ truncated Fourier rectangular matrix defined as follows

$$
\begin{aligned}
F_{K, L}(k, l) & =\frac{1}{\sqrt{K}} \exp \left(-j \frac{2 \pi k l}{K}\right), 0 \leq k \\
& <K \text { and } 0 \leq l<L
\end{aligned}
$$

In the context of a real transmission, a first estimation $\tilde{\boldsymbol{h}}_{x}(m)$ of $\boldsymbol{h}_{x}(m)$ can be proceeded through the classical least square as given in the following equation

$$
\tilde{h}_{x}(m)=\left(\begin{array}{c}
y_{0}(m) / x_{0} \\
\vdots \\
y_{K-1}(m) / x_{K-1}
\end{array}\right)
$$

As components of $\tilde{\boldsymbol{h}}_{x}(m)$ have not been averaged over a great number of observations, they are highly imprecise and they depend on the additive noise over the $y_{k}(m)$ received samples. A smoothing operation [15], leading to a new estimated $\hat{h}_{x}(m)$ vector, can be proposed through the following equation

$$
\hat{h}_{x}(m)=F_{K, L} F_{K, L}^{H} \hat{h}_{x}(m)
$$

This estimation algorithm is known as the indirect estimation [16,17], and some papers [18] propose to enhance it through the introduction of a noise power estimation and an adaptive weight, able to take this estimation into consideration.

The main drawback of this algorithm comes from Equation 41 that involves knowledge of all transmitted symbols $\left\{x_{k}\right\}_{k \in[0, K-1]}$. In a real transmission, only pilot symbols are known. If we consider that we have $K^{\prime}<K$ comb pilots $\left\{x_{0}, x_{1}, \ldots, x_{K^{\prime}}\right\}$, then Equation 41 becomes

$$
\tilde{h}^{\prime}{ }_{x}(m)=\left(\begin{array}{c}
y_{0},(m) / x_{0}, \\
\vdots \\
y_{K^{\prime}-1}(m) / x_{K^{\prime}-1}
\end{array}\right)
$$

where $\tilde{\boldsymbol{h}}^{\prime}{ }_{x}(m)$ is a $\left(K^{\prime} \times 1\right)$ vector representing the first estimation of the propagation channel frequency responses on the pilot locations.

The first estimation for the propagation channel impulse response can then be obtained through

$$
\tilde{a}_{x}(m)=F_{K^{\prime}, L}^{H} \tilde{h}^{\prime}{ }_{x}(m)
$$

Then, an interpolation step is required. It is performed by the following equation

$$
\hat{h}_{x}(m)=F_{K^{\prime}, L}^{H} \tilde{h}_{x}{ }_{x}(m)
$$

Equation 45 is devoted to the $m$ th antenna, and it can be generalized to all antennas. If we consider now the $k$ th component $\hat{h}_{x, k}(m)$ of all these $\left\{\hat{\boldsymbol{h}}_{x}(m)\right\}_{m \in[0, M-1]}$ vectors, we can introduce the $\hat{\boldsymbol{h}}_{x, k}$ vector defined as follows

$$
\hat{h}_{x, k}=\left(\begin{array}{c}
\hat{h}_{x, k}(0) \\
\vdots \\
\hat{h}_{x, k}(M-1)
\end{array}\right)
$$

The estimation $\hat{\boldsymbol{w}}_{h, k}$ of the $\boldsymbol{w}_{h, k}$ vector is then directly given by 


$$
\hat{w}_{h, k}=\frac{\hat{h}_{x, k}}{\hat{h}_{x, k}^{H} \hat{h}_{x, k}}
$$

\subsection{Estimation algorithm for $w_{z, k}$}

The $\boldsymbol{w}_{z, k}$ vector is jointly dependent on interference, and propagation channels are devoted to interference sources cancellation. It is well known that a $M$ antenna spatial filter is able to cancel $U=M-1$ interferers. In our particular case, where we choose $M=2$, we have then to cope with a unique interferer. In the sequel of this section, the $u$ index that represents the interferer index will be omitted in equations.

We consider the transmission of a unique OFDM symbol, the eigendecomposition of $\boldsymbol{R}_{z z, k}$ is given by

$$
R_{z z, k}=U_{k}\left(\begin{array}{cc}
\mu_{0, k} & 0 \\
0 & 0
\end{array}\right) U_{k}^{H}
$$

And the eigendecomposition of $\boldsymbol{C}_{z z, k}$ is given as follows

$$
C_{z z, k}=U_{k}\left(\begin{array}{cc}
0 & 0 \\
0 & \mu_{0, k}
\end{array}\right) U_{k}^{H}=\mu_{0, k} u_{2, k} u_{2, k}^{H}
$$

where $\boldsymbol{\mu}_{2, k}$ is the second column vector of $\boldsymbol{U}_{k}$ orthogonal to the interference vector $\left(\begin{array}{l}h_{z, k}(0) \\ h_{z, k}(1)\end{array}\right)$. Therefore, the following scalar vector is null $\boldsymbol{u}_{2, k}^{T}\left(\begin{array}{l}h_{z, k}(0) \\ h_{z, k}(1)\end{array}\right)=0$. Therefore, $\boldsymbol{u}_{2, k}=\alpha\left(\begin{array}{c}h_{z, k}(1) \\ -h_{z, k}(0)\end{array}\right)=0$, where $\alpha$ is a complex scalar. In the sequel, we set $\alpha=1$.

The filter $\boldsymbol{w}_{z, k}$ will then be rewritten as

$$
w_{z, k}=\frac{\mu_{0, k} u_{2, k} u_{2, k}^{H} h_{x, k}^{*}}{\mu_{0, k} h_{x, k}^{T} u_{2, k} u_{2, k}^{H} h_{x, k}^{*}}=\frac{u_{2, k}}{h_{x, k}^{T} u_{2, k}}
$$

Finally, the solution is given by

$$
w_{z, k}=\frac{w_{z, k, n}}{w_{z, k, d}}
$$

with

$$
w_{z, k, n}=\left(\begin{array}{c}
h_{z, k}(1) \\
-h_{z, k}(0)
\end{array}\right)
$$

and

$$
w_{z, k, d}=h_{z, k}(1) h_{x, k}(0)-h_{z, k}(0) h_{x, k}(1)
$$

The positive scalar $\rho_{k}$ in the general formula of the optimal spatial filter given in Equation 34 becomes

$$
\rho_{k}=\mu_{0, k} \frac{u_{2, k}^{H} h_{x, k}^{2}}{h_{x, k}^{2}}
$$

Therefore, $\rho_{k}$ is the intercorrelation factor between the desired and the interference channel vectors. From the theorem stated above and Equation 50, we emit the following proposition.

Proposition: In the case of two-antenna SIMO transmission disturbed by an interferer, the optimum combiner $\boldsymbol{w}_{k}$ is a weighted combination of the interference cancellation filter $\boldsymbol{w}_{z, k}$ and the maximum ratio combining filter $\boldsymbol{w}_{h, k}$.

The estimation of $\boldsymbol{w}_{z, k, n}$ and $w_{z, k, d}$ is a complex task that involves the knowledge of the desired and interferer propagation channels. Nevertheless, the expression of $\boldsymbol{w}_{z, k, n}$ and $w_{z, k, d}$ given by Equations 52 and 53, respectively, gives opportunities to project these components on a reduced Fourier basis.

\subsection{1 $w_{z, k, n}$ estimation}

On the first hand, we can notice that the components of $\boldsymbol{w}_{z, k, n}$ are simply those of the frequency response of the interferer propagation channels, corresponding to the $k$ th subcarrier. Therefore, the components of this filter can then easily be expressed on a reduced Fourier basis. For that purpose, we introduce the $(K \times 1), \boldsymbol{h}_{z}(m)$ vector that represents the frequency response of the propagation channel between the interferer and the $m$ th antenna as follows

$$
h_{z}(m)=\left(\begin{array}{c}
h_{z, 0}(m) \\
\vdots \\
h_{z, K-1}(m)
\end{array}\right)
$$

As in the previous section, the $\boldsymbol{h}_{z}(m)$ vector is linked to the $L$ taps impulse response $\boldsymbol{a}_{z}(m)$ through the following equation

$$
h_{z}(m)=F_{K, L} a_{z}(m)
$$

where the $(L \times 1) \boldsymbol{a}_{z}(m)$ vector is defined as follows

$$
a_{z}(m)=\left(\begin{array}{c}
a_{z, 0}(m) \\
\vdots \\
a_{z, L-1}(m)
\end{array}\right)
$$

Therefore, $\boldsymbol{w}_{z, n}$ can be expressed as

$$
w_{z, n}=F_{K, L} v_{z, n}
$$

\subsection{2 $w_{z, k, d}$ estimation}

On the other hand, we can notice that the $w_{z, k, d}$ is a scalar obtained by the product of two frequency response terms. It can then be viewed as the Fourier transform of the convolution of two impulses responses of propagation channels, and it can then be linked to a virtual $2 L$ taps impulse response. 
If we introduce the $(2 L \times 1) \boldsymbol{v}_{z, d}$ vector representing this virtual impulse response

$$
v_{z, d}=\left(\begin{array}{c}
v_{z, 0, d} \\
\vdots \\
v_{z, 2 L-1, d}
\end{array}\right)
$$

Then we can introduce the $\boldsymbol{w}_{z, d}$ vector defined as

$$
w_{z, d}=\left(\begin{array}{c}
w_{z, 0, d} \\
\vdots \\
w_{z, K-1, d}
\end{array}\right)
$$

with

$$
w_{z, d}=F_{K, 2 L} v_{z, d}
$$

\subsubsection{Replica spatial filter structure}

The decomposition of $\boldsymbol{w}_{z, k}$ in a numerator part and a denominator part as given by Equation 51 leads to propose a new spatial filter structure having two weights, represented by $\boldsymbol{w}_{z, k, n}$ acting over the received signal and a weight, represented by $w_{z, k, d}$ acting over the useful signal (Figure 4).

We can then introduce the error $e_{k}$ defined by

$$
e_{k}=w_{z, k, d} x_{k}-\left[y_{k}(0) \quad y_{k}(1)\right] w_{z, k, n}
$$

At this stage, knowing that the $\boldsymbol{w}_{z, k}$ filter has to cancel the interference, we can propose to identify its two components through an error square minimization criterion

$$
w_{z, k}=\arg \min _{w_{z, k, n}, w_{z, k, d}}\left|e_{k}\right|^{2}
$$

We have then to insert a constraint $\psi\left(\boldsymbol{w}_{z, k, n}, w_{z, k, d}\right)$ in order to avoid the trivial solution: $\left(\boldsymbol{w}_{z, k, n}=0, w_{z, k, d}=0\right)$. Moreover, the minimization has to be done over all frequencies. It is then necessary to propose a global criterion. For that purpose, we introduce the $\boldsymbol{X}$ transmitted diagonal data matrix, where each element $x_{k}$ corresponds to the desired symbol transmitted over the $k$ th subcarrier

$$
X=\operatorname{diag}\left\{x_{0}, x_{1}, \ldots, x_{K-1}\right\}
$$

We introduce also the bi-diagonal matrix $Y$ of the received signal over the two antennas

$$
Y=\left(\begin{array}{cccccc}
y_{0}(0) & 0 & 0 & y_{0}(1) & 0 & 0 \\
0 & \ddots & 0 & 0 & \ddots & 0 \\
0 & 0 & y_{K-1}(0) & 0 & 0 & y_{K-1}(1)
\end{array}\right)
$$

The $\boldsymbol{e}=\left(\begin{array}{c}e_{0} \\ \vdots \\ e_{K-1}\end{array}\right)$ vector representing errors over all subcarriers is then given by

$$
e=X w_{z, d}-Y w_{z, n}
$$

The filter weights are then given by the following minimization

$$
w_{z}=\underset{w_{z, d}, w_{z, n}}{\arg \min }\left(e^{2}-\mu \psi\left(w_{z, n}, w_{z, d}\right)\right)
$$

where $\mu$ is a Lagrange multiplier.

In $[12,19]$ and in a similar context, a constraint called maximum signal to interference plus noise constraint (MSINRC) is proposed. It is defined as

$$
\psi\left(w_{z, n}, w_{z, d}\right)=\left\|X w_{z, d}\right\|^{2}-1
$$

Without loss of generality, we can consider that all transmitted symbols $x_{k}$ are normalized: $\left|x_{k}\right|^{2}=1$, we have then $\boldsymbol{X}^{H} \boldsymbol{X}=\boldsymbol{I}$. The constraint presented in Equation 68 is then equivalent to $\left\|\boldsymbol{w}_{z, d}\right\|^{2}=1$.

By nulling the partial derivative of $\|\boldsymbol{e}\|^{2}-\mu \psi\left(\boldsymbol{w}_{z, n}, \boldsymbol{w}_{z, d}\right)$ with respect to $\boldsymbol{w}_{z, n}$ and $\boldsymbol{w}_{z, d}$ [20], we obtain the following system of equations

$$
\left\{\begin{array}{l}
X^{H} Y w_{z, n}-w_{z, d}=\mu w_{z, d} \\
w_{z, n}=\left(Y^{H} Y\right)^{-1} Y^{H} X w_{z, d}
\end{array}\right.
$$

Merging the two equations of Equation 68, we arrive to

$$
X^{H}\left(Y\left(Y^{H} Y\right)^{-1} Y^{H}-I\right) X w_{z, d}=\mu w_{z, d}
$$

It appears then that $\boldsymbol{w}_{z, d}$ is the eigenvector of the $\boldsymbol{X}^{H}\left(\boldsymbol{Y}\left(\boldsymbol{Y}^{H} \boldsymbol{Y}\right)^{-1} \boldsymbol{Y}^{H}-\boldsymbol{I}\right) \boldsymbol{X}$ matrix corresponding to the $\mu$ eigenvalue.

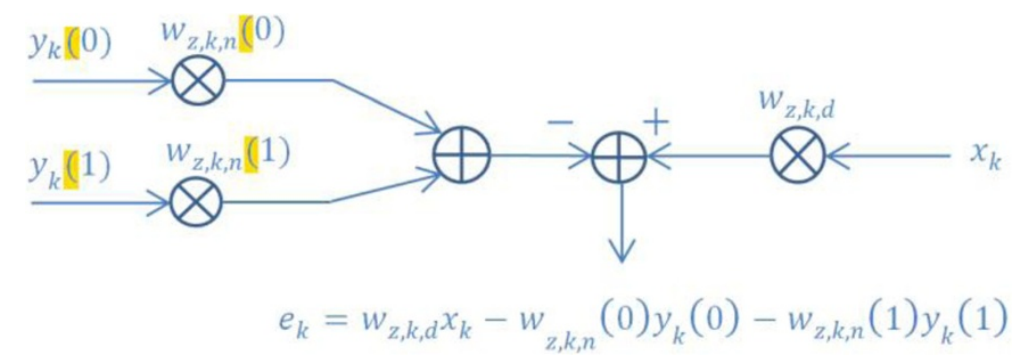

Figure 4 Replica spatial filter structure for the $k$ th subcarrier. 
Left multiplying the two sides of Equation 70 by $\boldsymbol{w}_{z, d}^{H}$, we obtain

$$
w_{z, d}^{H} X^{H}\left(Y\left(Y^{H} Y\right)^{-1} Y^{H}-I\right) X w_{z, d}=\mu w_{z, d}^{H} w_{z, d}
$$

This last equation leads to

$$
\frac{1}{\mu}=\frac{w_{z, d}^{H} w_{z, d}}{w_{z, d}^{H} X^{H}\left(Y\left(Y^{H} Y\right)^{-1} Y^{H}-I\right) X w_{z, d}}
$$

In the right side of Equation 72, we recognize the SINR formula; we can then conclude that

$$
\frac{1}{\mu}=\mathrm{SINR}
$$

Finally, as we have to maximize the SINR at the output of the filter, $\boldsymbol{w}_{z, d}$ has to be the generalized eigenvector which corresponds to the minimal eigenvalue $\mu$. Using Equation 61, Equation 72 becomes

$$
X^{H}\left(Y\left(Y^{H} Y\right)^{-1} Y^{H}-I\right) X F_{K, 2 L} v_{z, d}=\mu F_{K, 2 L} v_{z, d}
$$

Left multiplying the two sides of this equation by $\boldsymbol{F}_{K, 2 L}^{H}$ yields to

$$
F_{K, 2 L}^{H} X^{H}\left(Y\left(Y^{H} Y\right)^{-1} Y^{H}-I\right) X F_{K, 2 L} v_{z, d}=\mu v_{z, d}
$$

It appears that from Equation 74, the virtual impulse response $V_{z, d}$ is the $(2 L \times 1)$ eigenvector of the matrix $\boldsymbol{F}_{K, 2 L}^{H} \boldsymbol{X}^{H}\left(\boldsymbol{Y}\left(\boldsymbol{Y}^{H} \boldsymbol{Y}\right)^{-1} \boldsymbol{Y}^{H}-\boldsymbol{I}\right) \boldsymbol{X} \boldsymbol{F}_{K, 2 L}$ corresponding to the minimal eigenvalue $\mu$. This result is known and used by many authors. An enhanced maximum signal to interference plus noise constraint (EMSINRC) is proposed in [13]. It is based on the exploitation of the set $\boldsymbol{V}_{z, d}$ of all the eigenvectors of the previous matrix defined as

$$
V_{z, d}=\left[\begin{array}{lll}
v_{z, d}^{0} & \cdots & v_{z, d}^{2 L-1}
\end{array}\right]
$$

The EMSINRC algorithm introduces a linear combination of elements of $V_{z, d}$, in order to propose a composite virtual impulse response $v_{z, d}^{c}$ defined as follows

$$
v_{z, d}^{c}=\sum_{i=0}^{2 L-1} \eta_{i} \frac{1}{\mu_{i}} v_{z, d}^{i}
$$

where $\mu_{i}$ is the eigenvalue corresponding to the eigenvector $\boldsymbol{v}_{z, d}^{i}$. The complex term $\eta_{i}$ is defined such that $\eta_{i}=\arg \max _{\eta_{i}}\left\|\boldsymbol{v}_{z, d}^{c}\right\|^{2}$ and $\left|\eta_{i}\right|=1$. This complex scalar is acting as a phase term that aligns all eigenvectors.

Concerning the vector $\boldsymbol{v}_{z, n}$, it is obtained by merging Equation 58 into the second equation of the system (69). Hence, $\boldsymbol{v}_{z, n}$ is given as follows

$$
v_{z, n}=F_{K K, L L}^{H}\left(Y^{H} Y\right)^{-1} Y^{H} X F_{K K, L L} v_{z, d}
$$

$\boldsymbol{v}_{z, n}$ can then directly be obtained once $\boldsymbol{v}_{z, d}$ is determined.

Finally, having $\boldsymbol{w}_{z, d}$ and $\boldsymbol{w}_{z, n}$ form $\boldsymbol{v}_{z, d}$ and $\boldsymbol{v}_{z, n}$ by performing Equations 61 and 58, respectively, the interferer cancellation filter $\boldsymbol{w}_{z, k}=\frac{\boldsymbol{w}_{z, k, n}}{\boldsymbol{w}_{z, k, d}}$ for all subcarriers is obtained.

At this stage, all elements have been established and the optimal filter, given by Equation 34, can be estimated. The combination of Equation 34 is based on $\rho_{k}$ that can be obtained directly from Equation 54 noticing that the interferer power $\mu_{0}$ and $\sigma_{n}^{2}$ can be obtained through a noise plus interferer power estimation.

\section{Interference cancellation filter simplification}

The estimation of $\boldsymbol{w}_{z, d}$ and $\boldsymbol{w}_{z, n}$ involves an eigendecomposition which is a complex task, especially if we consider a practical implementation of the algorithm in the real-time context of the receiver. We will show, in this section, how this eigendecomposition can be avoided without involving any loss in the global performances of the algorithm.

The key element comes from the constraint introduced to avoid the trivial solution. Instead of $\left\|\boldsymbol{w}_{z, d}\right\|^{2}=1$, we propose to introduce a constraint directly applied to the virtual impulse response $\boldsymbol{v}_{z, d}$. This constraint consists in forcing a component of this vector to be equal to 1 .

If we denote by $k_{b}$, the index of this component, the constraint will be then given by $\boldsymbol{v}_{z, d}\left(k_{b}\right)=1$.

We will introduce now a square matrix $B$, with all zero components except a diagonal component set to 1 ; the constraint is then formalized as follows

$$
B v_{z, d}=1_{k_{b}}
$$

where $\mathbf{1}_{k_{b}}$ is a vector whose all components are null except $k_{b}^{t h}$ component set to 1 . The constraint can then be formalized with the following function $\psi^{\prime}\left(\boldsymbol{v}_{z, d}\right)$

$$
\psi^{\prime}\left(v_{z, d}\right)=\left\|B v_{z, d}\right\|^{2}-1
$$

The interference cancellation filter weights are then given by the new following minimization

$$
\left(v_{z, n}, v_{z, d}\right)=\arg \min _{v_{z, n}, v_{z, d}}\left(\|e\|^{2}-\mu^{\prime} \psi^{\prime}\left(v_{z, d}\right)\right)
$$

The derivation of Equation 80 with respect to $\boldsymbol{v}_{z, d}$ leads to the new solution $\left(v_{z, n}^{\prime}, v_{z, d}^{\prime}\right)$ given by

$$
v_{z, d}^{\prime}-F_{K, 2 L}^{H} X^{H} Y F_{K K, L L} v_{z, n}^{\prime}-\mu^{\prime} B^{H} B v_{z, d}^{\prime}=0
$$

Due to its structure, we have $\boldsymbol{B}^{H} \boldsymbol{B}=\boldsymbol{B}$. Merging this equation and Equation 78 in Equation 81 leads to 


$$
v_{z, d}^{\prime}-F_{K, 2 L}^{H} X^{H} Y F_{K K, L L} v^{\prime} z, n-\mu 1_{k_{b}}=0
$$

The derivation of Equation 80 with respect to $\boldsymbol{v}_{z, n}$ leads to

$$
-F_{K K, L L}^{H} Y^{H} X F_{K, 2 L} v_{z, d}^{\prime}+F_{K K, L L}^{H} Y^{H} Y F_{K K, L L} v_{z, n}^{\prime}=0
$$

By developing Equation 83, we obtain

$$
v_{z, n}^{\prime}=\left(F_{K K, L L}^{H} Y^{H} Y F_{K K, L L}\right)^{-1} F_{K K, L L}^{H} Y^{H} X F_{K, 2 L} v_{z, d}^{\prime}
$$

Merging Equation 84 in Equation 82, we obtain

$$
F_{K, 2 L}^{H} X^{H}\left(I-Y\left(Y^{H} Y\right)^{-1} Y^{H}\right) X F_{k, 2 L} v_{z, d}^{\prime}=\mu^{\prime} 1_{k_{b}}
$$

This last equation can be rewritten as

$$
v_{z, d}^{\prime}=\mu^{\prime} \Omega^{-1} 1_{k_{b}}
$$

with $\Omega=\boldsymbol{F}_{K, 2 L}^{H} \boldsymbol{X}^{H}\left(\boldsymbol{I}-\boldsymbol{Y}\left(\boldsymbol{Y}^{H} \boldsymbol{Y}\right)^{-1} \boldsymbol{Y}^{H}\right) \boldsymbol{X} \boldsymbol{F}_{K, 2 L}$ and where $\mu^{\prime}$ is a scalar chosen such that $\boldsymbol{v}_{z, d}^{\prime}\left(k_{b}\right)=1$.

Finally, the solution obtained from the proposed constraint leads to a solution given by Equations 84 and 86 . With these two equations, the eigendecomposition is avoided and the implementation complexity is widely reduced. The question of the optimality of this new solution has then to be analyzed in details. For that purpose, we propose to decompose the vector $\boldsymbol{v}_{z, d}^{\prime}$ in the $V_{\mathrm{z}, \mathrm{d}}$ orthogonal basis introduced in Equation 75 . We can then define a $(2 L \times 1)$ vector $\boldsymbol{\alpha}_{d}$ representing the image of $\boldsymbol{v}_{z, d}^{\prime}$ in the $\boldsymbol{V}_{\mathrm{z}, \mathrm{d}}$ basis as hereafter

$$
v_{z, d}^{\prime}=V z, d \alpha d
$$

As $V_{z, d}$ are eigenvectors of the $\Omega$ matrix, we have

$$
\Omega v_{z, d}^{\prime}=V_{z, d} I_{\mu} \alpha_{d}
$$

where $\boldsymbol{I}_{\mu}$ is the diagonal matrix of eigenvalue of $\boldsymbol{\Omega}$.

Considering Equation 86, the $\boldsymbol{\alpha}_{d}$ vector is then given by

Table 1 OFDM parameters

\begin{tabular}{lll}
\hline Symbol & Name & Value \\
\hline$K$ & Number of subcarriers & 32 \\
$N_{\text {FFT }}$ & FFT size & 32 \\
$N_{C D}$ & Cyclic prefix & $\frac{N_{\text {FFT }}}{8}=4$ \\
$W$ & Total bandwidth & $100 \mathrm{kHz}$ \\
$\Delta f$ & Subcarrier spacing & $3,125 \mathrm{kHz}$ \\
$T_{\text {OFDM }}$ & OFDM symbol duration & $360 \mu \mathrm{s}$ \\
$T_{\text {Frame }}$ & Frame duration & $18.36 \mathrm{~ms}$ \\
\hline
\end{tabular}

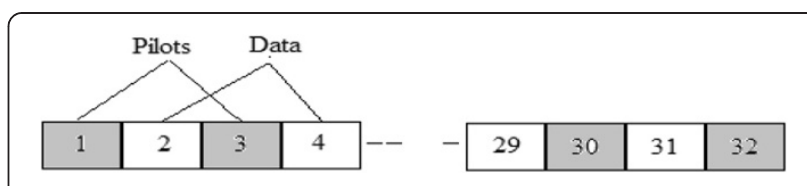

Figure 5 Pilots distribution over the OFDM symbol.

$$
\alpha_{d}=I_{\mu}^{-1} V_{z, d}^{H} \mu^{\prime} 1_{k_{b}}
$$

Finally, Equation 89 becomes

$$
v^{\prime}{ }_{z, d} \sum_{i=0}^{2 L-1} \eta^{\prime} i \frac{1}{\mu_{i}} v_{z, d}^{i}
$$

where $\eta^{\prime}{ }_{i}$ is the $i$ th component of the $k_{b}^{\text {th }}$ column vector of the matrix $V_{z, d}^{H}$.

This last equation has to be compared to Equation 76 related to the EMSINRC algorithm. It leads to the conclusion that the EMSINRC algorithm is in fact equivalent to a constraint modification leading to a new constraint given by Equation 79 .

\section{Simulation results}

In this section, we first evaluate performance of the various solutions presented in Sections 4 and 5. We will then compare:

- The solution based on $\left(\boldsymbol{v}_{z, n}, \boldsymbol{v}_{z, d}\right)$ that will be referred as maximum signal to interference plus noise ratio constraint (MSINRC)

- The solution based on $\left(v_{z, n}^{c}, v_{z, d}^{c}\right)$ that will be referred as enhanced maximum signal to interference plus noise ratio constraint (EMSINRC)

- The solution based on $\left(\boldsymbol{v}_{z, n}^{\prime}, \boldsymbol{v}_{z, d}^{\prime}\right)$ that will be referred as coefficient constraint (CC)

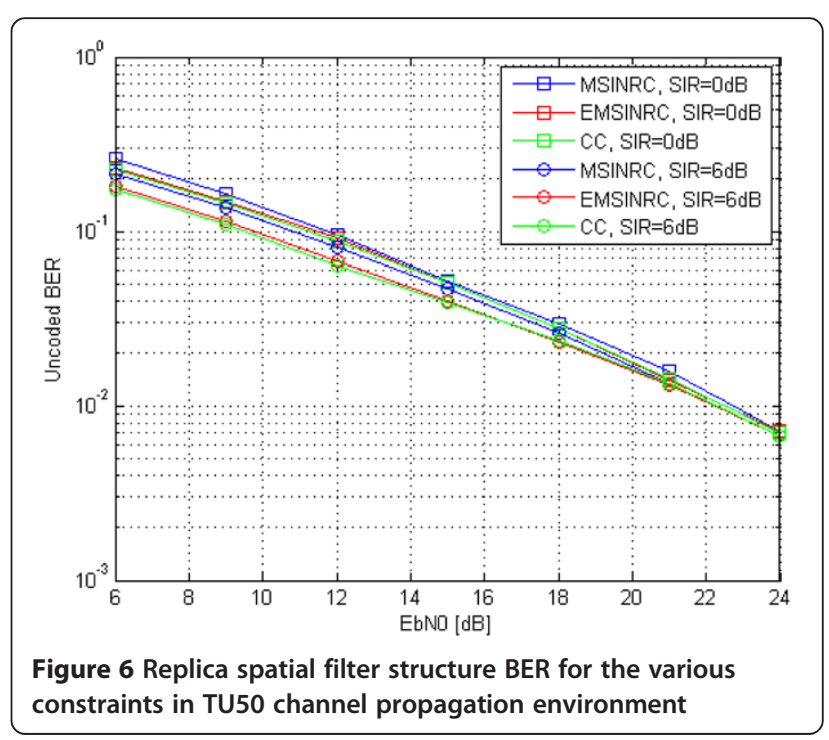




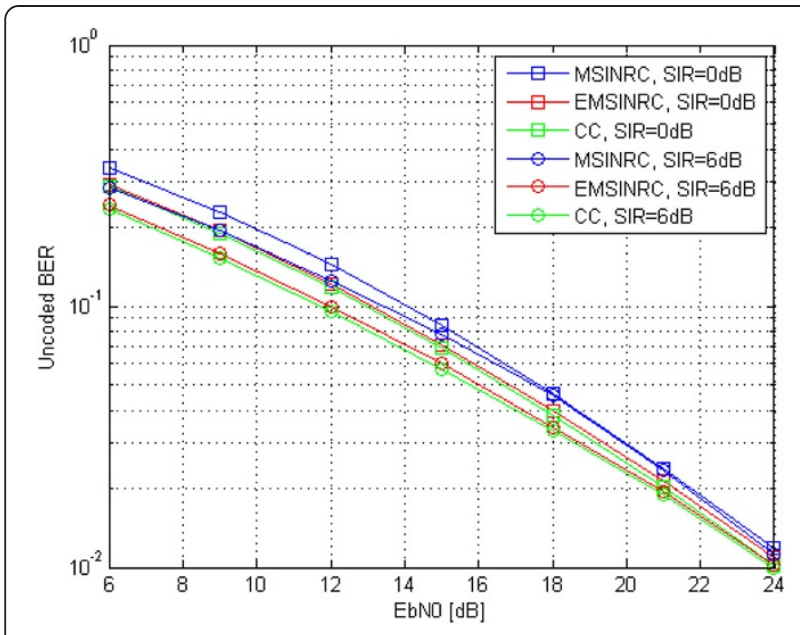

Figure 7 Replica spatial filter structure BER for the various constraints in HT200 channel propagation environment.

The simulation parameters based on OFDM waveform are summarized in Table 1, hereafter.

The modulation used is 4-QAM with the same power for pilots and data. The propagation channels used follow the Jakes model [21] with a propagation channel impulse response described in GSM standard [22], namely TU50 (typical urban with a mobile velocity of $50 \mathrm{~km} / \mathrm{h}$ ) and HT200 (hilly terrain with a mobile velocity of $200 \mathrm{~km} / \mathrm{h}$ ). The pilots are uniformly distributed along the frequency axis with a rate of $\frac{1}{2}$ as depicted in Figure 5 and along the time axis with a rate of $\frac{1}{5}$.

Algorithm's weights are estimated on the plots position for each OFDM symbol containing pilots. An interpolation within the subspace generated by the reduced DFT $\boldsymbol{F}_{L}$ and

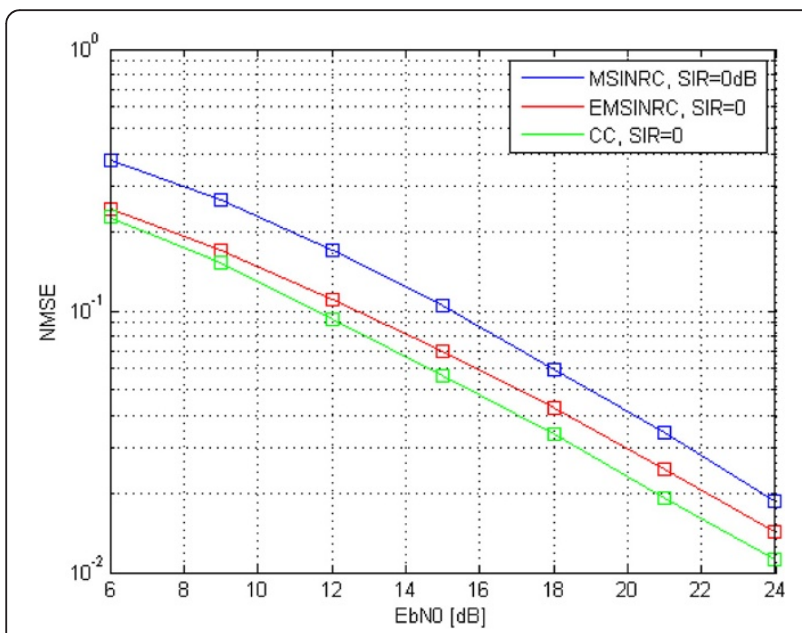

Figure 8 Replica spatial filter structure NMSE of $v$ for various constraints in TU50 channel propagation environment.

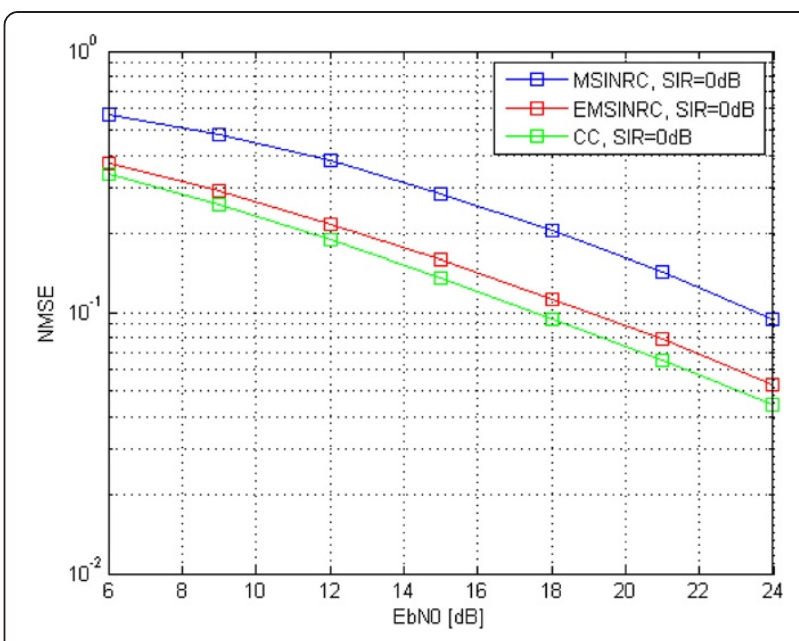

Figure 9 Replica spatial filter structure NMSE of $v$ for various constraints in HT200 channel propagation environment.

$\boldsymbol{F}_{2 L}$ is followed to extend the weights to the whole OFDM symbol.

However, along the time axis, the interpolation method used is the spline cubic [23].

Performance assessment is performed by comparing the uncoded BER (bit error rate before channel decoding) vs. $E_{b} N_{0}$ (signal to noise ratio) at signal to interference ratio (SIR) equal to 0 and $6 \mathrm{~dB}$. As in $[24,25]$, an additional comparison is done to confirm the previous one and consists on the study of the normalized mean square error (NMSE) of $\hat{v}$ expressed as NMSE $=\frac{\|\hat{v}-v\|^{2}}{\|v\|^{2}}$, where $v$ is the exact weight vector cancelling totally the interference, and $\hat{\boldsymbol{v}}=\left[\begin{array}{l}\hat{\boldsymbol{v}}_{n} \\ \hat{\boldsymbol{v}}_{d}\end{array}\right]$ is the estimation of $\boldsymbol{v}$ using the constraints studied in Section 4.

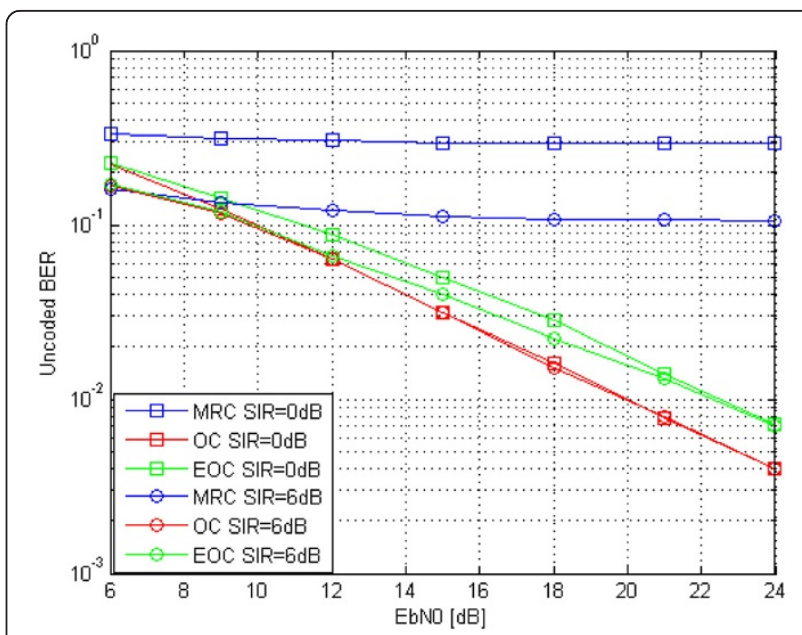

Figure 10 BER performance of EOC vs. MRC and OC in TU50 channel propagation environment. 


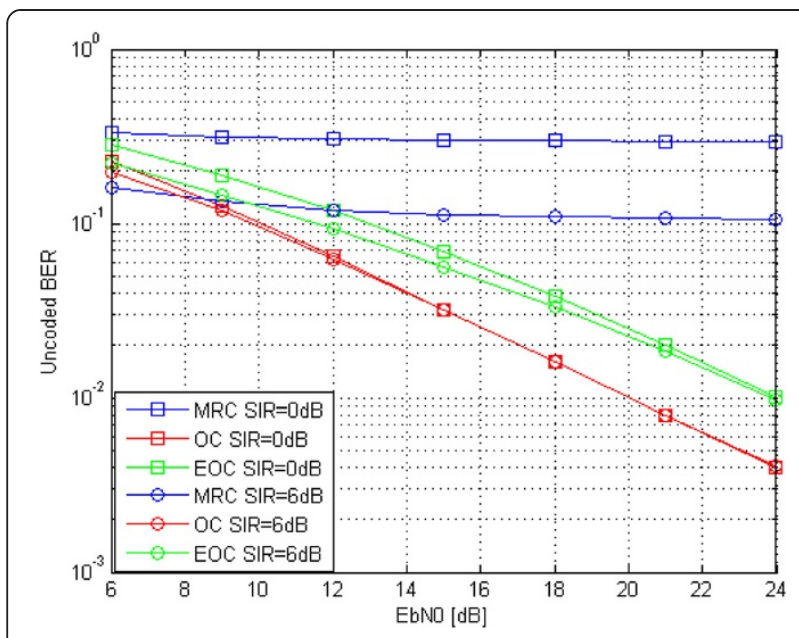

Figure 11 BER performance of EOC vs. MRC and OC in HT200 channel propagation environment.

Figures 6 and 7 depict the uncoded BER obtained for the different constraints used in the weight vector estimation $\hat{v}$. The observation of the curves confirms the theoretical analysis where the CC outperforms the MSINRC in the both cases of propagation channel environment. The difference in the performance is more pronounced for low $E_{b} N_{0}$ level, and obviously, the curves converge when $E_{b} N_{0}$ becomes high. Moreover, as expected in the theoretical analysis, the curves of CC and EMSINRC are superposed.

We conclude that $\mathrm{CC}$ is not only the best choice in the sense of bit error rate performance but also achieves very low complexity in its implementation. As shown in Equation 90, the solution is obtained by a simple matrix inversion unlike the other methods which need an eigendecomposition.

This difference of BER performance between the constraints studied in Section 5 is confirmed by the NMSE as depicted by Figures 8 and 9. It is clearly shown that for CC constraint, the NMSE is less important than the MSINRC and close to the EMSINRC.

In the sequel, we discuss the performance of the estimated optimum combiner (EOC) based on the weighted combination of the estimated replica spatial filter using $\mathrm{CC}$ and the estimated MRC. EOC is then compared to the exact MRC (propagation channel $h_{d}$ is perfectly

Table 2 Execution time and memory use

\begin{tabular}{lccc}
\hline Step & $\begin{array}{c}\text { Memory used } \\
\text { [bytes] }\end{array}$ & $\begin{array}{c}\text { DSP cycle } \\
\text { count }\end{array}$ & $\begin{array}{c}\text { Execution } \\
\text { time }(\boldsymbol{\mu} \mathbf{s})\end{array}$ \\
\hline $\begin{array}{l}\text { Weights estimation } \\
\text { (with Gauss) }\end{array}$ & 2,348 & $1,859,684$ & 1,860 \\
Gauss (with prescaling) & 420 & $84,770(97,205)$ & 84 \\
Cholesky (with prescaling) & 1,280 & $70,306(83,777)$ & 70 \\
Channel equalization & 28 & 458,725 & 458 \\
\hline
\end{tabular}

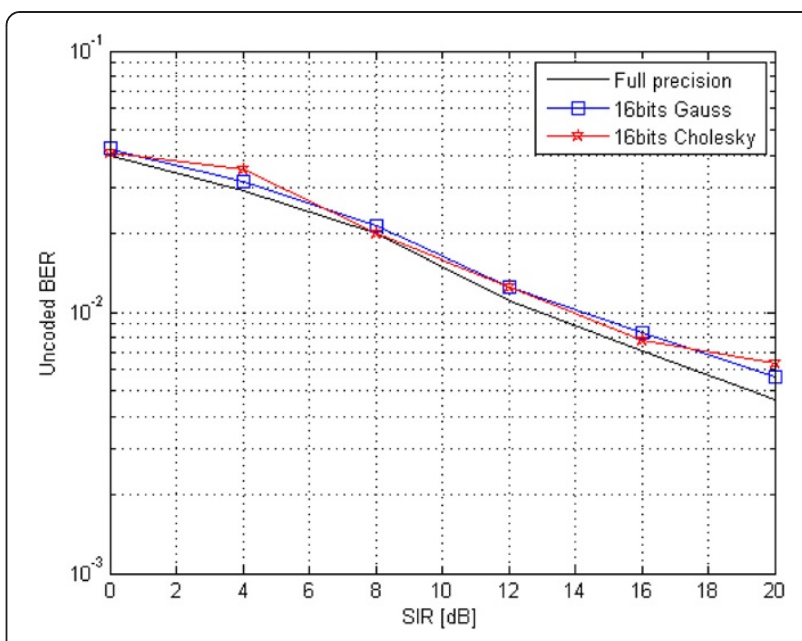

Figure 12 BER performance replica filter on 16-bit fixed point in TU50 channel propagation environment.

known) and the exact optimum combiner (OC) where the covariance matrix $R_{n n}$ and propagation channel $h_{d}$ are perfectly known. Furthermore, additive white Gaussian noise (AWGN) power is assumed known.

Figures 8 and 9 show that the EOC in TU50 propagation environment is better than in HT200 conditions. In TU50, the EOC presents a good performance comparing to $\mathrm{OC}$, and there is only $2 \mathrm{~dB}$ of difference at $E_{b} N_{0}=20 \mathrm{~dB}$. However, in HT200 environment, this difference is more significant and is approximately about $4 \mathrm{~dB}$ at $E_{b} N_{0}=20 \mathrm{~dB}$. This degradation is attributed to the estimation error of the EOC, which is proportional to the size of the weight vector. For example, in our case, the total weight vector length is equal to $4 L_{\mathrm{TU}}=8$ for TU50 and $4 L_{\mathrm{HT}}=12$ for HT200. We denote by $L_{\mathrm{TU}}=2$ and $L_{\mathrm{HT}}=3$ the maximum impulse response length of the propagation channels in TU50 and HT200 environments, respectively (Figures 10 and 11).

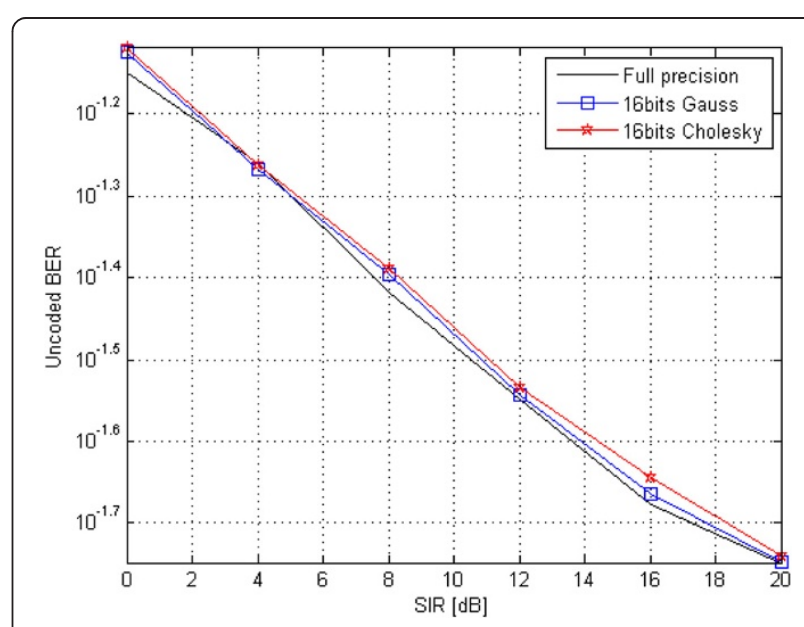

Figure 13 BER performance replica filter on 16-bit fixed point in HT200 channel propagation environment. 


\section{Real-time implementation}

The complexity of an algorithm is often quantified in terms of the number of arithmetic operations. This quantification provides an estimate of the use of memory and execution time necessary to execute the algorithm.

Generally, algorithms are designed for real-time use and have to be performed by finite precision processors then the study of complexity raised above can meet the first requirement, but not enough to satisfy the second. In this section, we have performed a 16-bit DSP implementation in order to insure a full analysis, namely to check that the real-time constraint and 16-bit fixed points degradation tolerance are respected.

EOC using CC constraint is implemented into the 16bit fixed-point DSP (TMS 320C6474) which works at frequency clock of $1 \mathrm{G} \mathrm{Hz}$ [26]. The whole of the receive chain is implemented, but we will show only the results of the part where the discussed algorithms are involved, namely the channel equalization.

Table 2 shows the execution time and memory uses performance of the algorithm. The main complexity cost in the EOC algorithm steels the relatively high dimension matrix to be inverted. Two methods of matrix inversion are implemented, namely Gauss-Jordan and Cholesky decomposition. The difference in the sense of execution time is negligible. The total execution time for the channel equalization is $210.7 \mu$ s per OFDM symbol which represents $1.15 \%$ of the frame duration.

The degradation caused by the 16-bit fixed-point format over the full precision floating point is shown in Figures 12 and 13 for TU50 and HT200 environments, respectively. These results are given in terms of uncoded BER vs. signal to interference ratio (SIR) at $E_{b} N_{0}=20 \mathrm{~dB}$. One can observe that we have obtained satisfactory results in both cases of Gauss and Cholesky implementation.

\section{Conclusions}

This paper presents a new expression for the optimum combining filter for the case of a two-antenna based receiver. This expression is a weighted combination of two components. The first component is the combiner obtained in the case of null additive white Gaussian noise and the second is the combiner obtained in the case of null interference. This decomposition is important because it allows the optimal combining estimation through two filters of known sizes, so the problem of the size of the filters is avoided. A detailed study dedicated to a SIMO transmission in the case of one interferer and two receive antennas is presented in order to confirm the importance of this decomposition. A DSP implementation is also investigated to quantify the algorithm complexity. The success of this implementation is a proof that this algorithm is ready to use at least in cases close to the given example.
We note that all developments presented in the estimation part of the OC study have been based on one dimension estimation, mainly the frequency axis of the OFDM frame. However, the proposed method can be done on the time axis of the frame such as in $[27,28]$ or on both axis.

Competing interests

The authors declare that they have no competing interests.

\section{Author details}

${ }^{1}$ CNAM/CEDRIC/Laetitia, Paris, France. ${ }^{2}$ Airbus Group, Elancourt, France.

Received: 28 March 2014 Accepted: 29 July 2014

Published: 15 August 2014

\section{References}

1. GG Raleigh, JM Cioffi, Spatio-temporal coding for wireless communication. IEEE Trans. Commun. 46, 357-366 (1998)

2. Z Tang, RC Cannizzaro, G Leus, P Banelli, Pilot-assisted time-varying channel estimation for OFDM systems. IEEE Trans. Signal Process. 55(5), 2226-2238 (2007)

3. AJ Baird, CL Zahm, Performance criteria for narrowband array processing. Proc. IEEE Conf. Decis. Control. 1, 564-565 (1971)

4. Y Zhihang, J MinChul, K II-Min, Outage probability and optimum combining for time division broadcast protocol. IEEE Trans. Commun. 10, 1362-1367 (2011)

5. JH Winters, Optimum combining in digital mobile radio with cochannel interference. IEEE J. Selected Areas Commun. 2, 528-539 (1984)

6. S Roy, P Fortier, Maximal-ratio combining architectures and performance with channel estimation based on a training sequence. IEEE Trans. Wireless Commun. 3, 1154-1164 (2004)

7. Y Hara, Weight-convergence analysis of adaptive antenna arrays based on SMI algorithm. IEEE Trans. Wireless Commun. 2, 56-57 (2003)

8. LL Horowitz, H Blatt, WG Brodsky, Controlling adaptive antenna arrays with the sample matrix inversion algorithm. IEEE Trans. Aerosp. Electron. Syst. 15, 840-848 (1979)

9. J Ketonen, Equalization and Channel Estimation Algorithms and Implementations for Cellular MIMO-OFDM Downlink (University Of Oulu, Oulu, Finland, 2012)

10. I Barhumi, G Leus, M Moonen, MMSE Estimation of Basis Expansion Models for Rapidly Time-Varying Channels (ESAT laboratory of the Katholieke Universiteit Leuven, Leuven, Belgium, 2005)

11. S-H Lee, H-S Kim, Y-H Lee, Complexity reduced space ML detection for other-cell interference mitigation in SIMO cellular systems. Eur. Trans. Telecom. 22(1), 51-60 (2011)

12. MA Lagunas, J Vidal, Al Pérez Neira, Joint array combining and MLSE for single-user receivers in multipath gaussian multiuser channels. IEEE J. Selected Areas Commun. 18(11), 2252-2259 (2000)

13. J Vidal, M Cabrera, A Augustin, Full exploitation of diversity in space-time MMSE receivers. IEEE Vehicular Tech. Conf. 5, 2497-2502 (2000)

14. R Maoudj, M Terre, Post-combiner for interference cancellation algorithm. Int. Conf. Softw. Telecomm. Comput. Netw. 1, 1-5 (2012)

15. C Dumard, T Zemen, Low-complexity MIMO multiuser receiver: a joint antenna detection scheme for time-varying channels. IEEE Trans. Signal Process. 56(7), 2931-2940 (2008)

16. P Hoeher, S Kaiser, P Robertson, Pilot-symbol-aided channel estimation in time and frequency. IEEE Global Telecom. Conf. GLOBECOM 90-96 (1997)

17. G Auer, E Karipidis, Pilot aided channel estimation for OFDM: a separated approach for smoothing and interpolation. IEEE Trans. Commun. 4, 2173-2178 (2005)

18. F Salman, J Cosmas, Y Zhang, Pilot aided channel estimation for SISO and SIMO in DVB-T2 (Annual post graduate symposium on the convergences of telecommunication, networking and broadcasting (Liverpool, England, 2012), pp. 1-6

19. M Caus, A Perez-Neira, Space-time receiver for filterbank based multicarrier systems, in Procedure of international ITG workshop on Smart Antennas WSA (Bremen, Germany, 2010), pp. 421-427

20. H Wen-Sheng, C Bor-Sen, ICI Cancellation for OFDM communication systems in time-varying multipath fading channels. IEEE Trans. Commun. 4(5), 2100-2110 (2005)

21. WC Jakes, Microwave Mobile Communications (John Wiley \& Sons Inc, Michigan, USA, 1975) 
22. COST-207, Digital land mobile radio communications (Final report of the COST-project 207, Commission Of the European Community, Brussels, 1989)

23. SA Dyer, JS Dyer, Cubic-spline interpolation. 1. IEEE Instrum. Meas. Mag. 4(1), 44-46 (2001)

24. MR Raghavendra, S Bhashyam, K Giridar, Interference rejection for parametric channel estimation in reuse-1 cellular OFDM systems. IEEE Trans. Vehicular Tech. 58, 4342-4352 (2009)

25. T Zemen, CF Mecklenbräuker, J Wehinger, RR Müller, Iterative joint time-variant channel estimation and multi-user detection for MC-CDMA. IEEE Trans. Commun. 5, 1469-1478 (2006)

26. TMS320C6474 Multicore digital signal processor data manual (Rev. H) (Texas Instruments Incorporated, 2001)

27. T Zemen, CF Mecklenbrauker, Time-variant channel estimation using discrete prolate spheroidal sequences. IEEE Trans. Signal Process. 53, 3597-3607 (2005)

28. T Zemen, L Bernado, N Czink, Iterative time-variant channel estimation for $802.11 \mathrm{p}$ using generalized discrete prolate spheroidal sequences. IEEE Vehicular Tech. Conf. 1, 1222-1233 (2012)

doi:10.1186/1687-6180-2014-127

Cite this article as: Maoudj et al.: Spatial filter decomposition for interference mitigation. EURASIP Journal on Advances in Signal Processing 2014 2014:127.

\section{Submit your manuscript to a SpringerOpen ${ }^{\circ}$ journal and benefit from:}

- Convenient online submission

- Rigorous peer review

- Immediate publication on acceptance

- Open access: articles freely available online

- High visibility within the field

- Retaining the copyright to your article 